\title{
Antecedentes Organizacionales y Personales de las Intenciones de Compartir Conocimiento: Apoyo, Clima y Compromiso con la Organización
}

\section{Organizational and Individual Antecedents on Knowledge- Sharing Intention: Perceived Organizational Support, Climate and Organizational Commitment}

\author{
Guillermina Tormo Carbó \\ Universitat Politècnica de València
}

Amparo Osca Segovia
UNED

\begin{abstract}
Resumen. El objetivo de esta investigación es analizar de forma exploratoria el papel de tres antecedentes organizacionales y personales en las intenciones de compartir conocimiento: el apoyo, el clima y el compromiso con la organización. La muestra está formada por 168 empleados del sector cerámico, y se ha puesto a prueba un modelo de ecuaciones estructurales que contempla relaciones directas e indirectas. En concreto se analizan las relaciones directas del apoyo y el clima (específicamente las dimensiones orientación a la tarea y apoyo a la innovación) sobre las intenciones de compartir conocimiento, y las relaciones indirectas de esas dos variables a través del compromiso afectivo de los empleados con su organización. Los resultados muestran que la orientación a la tarea tiene un efecto directo sobre las intenciones de compartir conocimiento, mientras que las otras dos dimensiones, el apoyo a la innovación y el apoyo organizacional, influyen de forma indirecta a través del compromiso afectivo. Al final se comentan los resultados desde un punto de vista aplicado.

Palabras clave: apoyo organizacional, clima psicológico, compromiso afectivo, intenciones de compartir conocimiento.
\end{abstract}

\begin{abstract}
The main goal of this study is to investigate the influence of three organizational and individual antecedents on knowledge-sharing intention: perceived organizational support, psychological climate and organizational commitment. To analyze the direct and indirect relationships among variables, a structural equations model is proposed. The object of analysis is, specifically, the direct relationship between organizational support and psychological climate (task orientation and support for innovation) on knowledge-sharing intentions and the indirect relationship of these antecedents through employees' affective commitment. Results, based on a survey of 168 employees in the tile industry, show that task orientation has only a direct effect on knowledge-sharing intentions, whereas the two dimensions related to support (support for innovation and organizational support) have an indirect effect on knowledge-sharing intention because they increase employees' affective commitment. This article ends with a discussion on the results from a practical point of view.

Keywords: affective commitment, knowledge sharing intentions, organizational support, psychological climate.
\end{abstract}

En el contexto económico actual el conocimiento es un recurso organizativo estratégico y la ventaja competitiva de las empresas depende en gran medida de cómo se gestione (p. ej. Gagné, 2009). En este marco, compartir el conocimiento con los miembros de la organización se revela como un proceso central para explicar la innovación (Camelo, Garcia y Sousa, 2010) y capacidad de aprendizaje de una organización (Foss, Husted y Michailova, 2010). Sin embargo, esta conducta no se suele producir de forma espontánea, por lo que se ha planteado la necesidad de investigar qué fac-

La correspondencia deberá enviarse a Amparo Osca. Facultad de Psicología (UNED), C/ Juan del Rosal, 10 Madrid 28040. Correo electrónico:aosca@psi.uned.es tores la explican (Wang y Noe, 2010). Así, el objetivo de esta investigación es analizar de forma exploratoria la influencia de tres antecedentes organizacionales y personales en las intenciones de compartir conocimiento: el apoyo, el clima y el compromiso con la organización (Figura 1). Dada la escasez de estudios similares en los que apoyar este trabajo y que las conductas de compartir conocimiento pueden considerarse conductas extra-rol (Yu y Chu, 2007), que responden a relaciones de intercambio social (Lester, Meglino y Korsgaard, 2008), a continuación se revisa la investigación que muestra el peso de estas tres variables en las actitudes hacia el trabajo y el desempeño, tanto en el que se refiere a las conductas propias del puesto, como al extra-rol. 


\section{Apoyo Organizacional}

La teoría del Apoyo Organizacional (Eisenberger, Huntington, Hutchison y Sowa, 1986) se basa en la norma de reciprocidad (Gouldner, 1960) para explicar que los empleados que se sienten apoyados por su organización, se muestran más comprometidos y hacen mejor su trabajo, pues al percibir que están en deuda, tienden a actuar de forma recíproca y a devolver lo que han recibido. Esto supone además que se implican con los objetivos de la organización y llevan a cabo conductas extra-rol de ayuda a sus compañeros (Eisenberger, Armeli, Rexwinkel, Lynch y Rhoades, 2001; Rhoades y Eisenberger, 2002).

La abundante investigación realizada sobre el papel de apoyo organizacional en los últimos años ha permitido que aparezcan diferentes metanálisis sobre el tema. Concretamente, el de Rhoades y Eisenberger (2002) muestra que el apoyo organizacional se relaciona significativamente con la satisfacción (.62), la implicación (.67), la intención de abandonar la organización (-.51) y el desempeño, tanto el que se refiere a las conductas propias del puesto (.18), como a las conductas extra-rol o de ciudadanía (.28). Por su parte, el metanálisis de Riggle, Edmondson y Hansen (2009) indica que el apoyo organizacional mantiene fuertes relaciones de carácter positivo con la satisfacción y la implicación con la organización; relaciones medias y también positivas con el desempeño y fuertes relaciones de carácter negativo con la intención de abandonar la organización. Estudios recientes van en la misma dirección y confirman la relación del apoyo con diferentes resultados organizacionales: la satisfacción con el trabajo (MacKain, Myers, Ostapiej y Newman, 2010), la propensión al abandono (Dawley, 2010; Edwards y Peccei, 2010; Filipova, 2011), el desempeño en el puesto (Alpkan, 2010; Darolia, Kumari y Darolia, 2010; Pazy y Ganzach, 2009; Shelton, Waite y Makela, 2010) y el extra-rol o las conductas de ciudadanía (Chen, Eisenberger, Johnson, Sucharski y Aselage, 2009; Liu, 2009; Tremblay, Cloutier, Simard, Chenevert y Vandenberghe, 2010; Wayne, Shore, Bommer y Tetrick, 2002; Webster y Adams, 2010). Concretamente y respecto al compromiso con la organización se observa que los empleados que se sienten más apoyados se muestran también más implicados y comprometidos. Dos estudios metanalíticos confirman que el apoyo se vincula positivamente con la implicación. El de Chiaburu y Harrison (2008) constata la influencia positiva del apoyo de los compañeros en las actitudes de los empleados hacia su organización y el de $\mathrm{Ng}$ y Sorensen (2008) muestra que el apoyo de supervisores y compañeros se relaciona con la implicación afectiva de los empleados (.48 y .28 , respectivamente). Otros trabajos más recientes obtienen resultados en la misma línea. Por ejemplo, Guerrero y Herrbach (2009) analizan los antecedentes de la implicación desde la teoría del intercambio social y observan que el apoyo que se presta a los empleados se vincula con la implicación que muestran hacia su organización. Rousseau y Aube (2010) también encuentran vínculos importantes entre el apoyo y la implicación, e identifican algunas variables contextuales y del puesto que modulan esas relaciones. Hi, Lai y Lu (2011) constatan que el apoyo organizacional se relaciona positivamente con la implicación de los empleados, sobre todo, el apoyo por parte de la dirección.

Sin embargo, todavía hay pocos estudios empíricos que analicen el papel del apoyo organizacional en el ámbito de la gestión del conocimiento. Para Kulkarni, Ravindran y Freeze (2006) los empleados que se sienten apoyados por su organización utilizan mejores estrategias para compartir lo que saben con sus compañeros. Cabrera, Collins y Salgado (2006), en un estudio con empleados de una empresa multinacional ubicada en España, identifican diferentes antecedentes organizacionales de las conductas de compartir conocimiento y el que más influye es el apoyo de supervisores y colegas. Por su parte, Bartol, Liu, Zeng y Wu (2009) observan que el apoyo aumenta la probabilidad de que se comparta lo que se sabe con los colegas de trabajo.

\section{Clima organizacional}

Respecto al papel del clima organizacional y dado que esta variable sirve como referencia para saber si un comportamiento resulta adecuado en un grupo u organización, es comprensible que aquellos empleados que creen que su organización valora sus ideas y reciben recursos para la innovación, se sientan más implicados y satisfechos y muestran un mejor desempeño. Algunos metanálisis sobre el papel del clima muestran que se relaciona significativamente con el compromiso y el desempeño (Parker et al, 2003). Específicamente, Carr, Schmidt, Ford y DeShon (2003) con 70 muestras $(\mathrm{N}=27123)$ y utilizando la taxonomía de Ostroff y Schmitt (1993), que distingue entre clima afectivo (caracterizado por la confianza, la calidez y las recompensas sociales), cognitivo (por la innovación, la autonomía y las recompensas intrínsecas) e instrumental (por el logro y las recompensas extrínsecas), obtienen que se relaciona con el compromiso de los empleados $(\mathrm{r}=.28$ para el clima cognitivo, $\mathrm{r}=.26$ para el instrumental y $r=.34$ para el afectivo).

Sin embargo, en las búsquedas bibliográficas realizadas sólo aparecen dos trabajos que relacionen el clima organizacional y las conductas de ciudadanía. Choi (2007) observa que el clima de innovación se vincula con aquellas conductas extra-rol que buscan hacer cambios constructivos en los métodos, procedimientos y normas de trabajo y D'Amato y Zijlstra (2008) muestran que un clima organizacional positivo es un antecedente de las conductas extra-rol. Por su parte, el metanálisis de Hulsheger, Anderson y Salgado (2009) con 104 estudios ( $\mathrm{N}=50096)$, señala que las dimensiones 
de clima, apoyo para la innovación y orientación a la tarea, se relacionan de forma importante con los comportamientos para la innovación $(.47$ y .42 , respectivamente). Si se analiza más específicamente la relación entre el clima y la gestión del conocimiento, se encuentra que Zarraga y Bonache (2003) en nuestro contexto, observan que un clima caracterizado por la confianza mutua entre sus miembros, orientado y abierto a la innovación y a las mejoras y en el que se valora el respeto, influye positivamente en que se comparta lo que se sabe con los compañeros. Fuera de nuestro contexto se observa también que el clima organizacional influye en la intención de compartir lo que se sabe con los compañeros (Lee y Choi, 2003), de forma que las dimensiones relacionadas con las interacciones, la apertura y la innovación influyen en la intención de compartir conocimiento (Lin y Lee, 2006).

\section{Compromiso con la organización}

El compromiso hace referencia a la fuerza con que un individuo se identifica y está involucrado con su organización (Allen y Meyer, 1996). Los empleados con altos niveles de compromiso se implican con los objetivos de la organización y actúan de forma consecuente, mejorando su desempeño y realizando conductas pro-sociales hacia sus colegas y la organización en su conjunto (Cropanzano y Mitchell, 2005). Riketta (2002) después de revisar 93 estudios encuentra una correlación significativa de .20 entre implicación con la organización y desempeño, aunque identifica tres variables que están influyendo, de forma que la relación es más fuerte cuando se evalúa el desempeño extra-rol $(\mathrm{r}=.25)$ que el desempeño en el puesto $(\mathrm{r}=.18)$. Además, según Ardichvili (2008) cuando el vínculo que une a los empleados con su organización es el compromiso afectivo, están dispuestos a realizar esfuerzos extra para actuar con ciudadanía compartiendo su conocimiento con sus compañeros. En este sentido, Lin (2007) obtiene en un estudio con alumnos taiwaneses que el compromiso afectivo es un factor fundamental para que compartan lo que saben. Resultados parecidos a los de Cabrera et al. (2006), quienes observan que el compromiso se relaciona positiva y significativamente con las conductas de compartir conocimiento, aunque este efecto desaparece cuando en el modelo propuesto se consideran otras variables. Golden y Raghuram (2010) también encuentran que el compromiso afectivo con la organización se relaciona positivamente con que los empleados (teletrabajadores) compartan su conocimiento con sus compañeros En la misma línea, Han, Chiang y Chang (2010), en un estudio en empresas de alta tecnología, encuentran relaciones entre el compromiso organizacional y las conductas de compartir conocimiento. En España, Camelo et al. (2010) en una muestra de 87 empresas innovadoras, observan que el compromiso afectivo es un factor motivacional para que se comparta lo que se sabe y actúa como mediador entre las prácticas de recursos humanos de alta implicación y esta variable.

En otros estudios también en el ámbito de la gestión del conocimiento, se ponen a prueba modelos más complejos que no sólo analizan relaciones directas. Por ejemplo, Tsai y Cheng (2010) obtienen que el clima influye en la intención de compartir conocimiento, pero a través de dos variables personales, o Bock, Zmud, Kim y Lee (2005) y Tohidinia y Mosakhani (2010), quienes encuentran que el clima influye positivamente en la intención de compartir conocimiento a través de su efecto sobre las normas organizacionales. Cabrera et al. (2006) observan que el compromiso está relacionado positiva y significativamente con las conductas de compartir conocimiento, aunque este efecto desaparece cuando en el modelo propuesto se consideran otras variables. Dado que sus resultados contradicen los de otras investigaciones previas, como la de Kalman (1999) que encuentra que el compromiso es clave para compartir conocimiento, estos autores recomiendan que se continúe investigando con modelos más complejos. Siguiendo toda esta argumentación, el estudio que se propone pone a prueba de forma exploratoria un modelo que analiza de forma conjunta y a través de ecuaciones estructurales, el papel del apoyo y el clima organizacional sobre las intenciones de compartir conocimiento de forma directa e indirecta, a través de su influencia sobre el compromiso afectivo de los empleados con su organización (Figura 1).

Concretamente, las hipótesis que se ponen a prueba son las siguientes:

Hipótesis 1: Se espera una relación positiva entre el apoyo organizacional y el compromiso con la organización.

Hipótesis 2: Se espera una relación positiva entre el apoyo organizacional y la intención de compartir conocimiento con los compañeros.

Hipótesis 3: Se espera una relación positiva entre el clima (dimensión orientación a la tarea) y el compromiso afectivo de los empleados.

Hipótesis 4: Se espera una relación positiva entre el clima (dimensión apoyo a la innovación) y el compromiso afectivo de los empleados.

Hipótesis 5: Se espera una relación positiva entre el clima (dimensión orientación a la tarea) y la intención de compartir conocimiento.

Hipótesis 6: Se espera una relación positiva entre el clima (dimensión apoyo a la innovación) y la intención de compartir conocimiento. 
Hipóteis 7: Se espera una relación positiva entre el compromiso afectivo y la intención de compartir conocimiento.

\section{Método}

\section{Participantes}

El estudio se ha realizado sobre una muestra de 168 empleados de 42 empresas medianas (de entre $50 \mathrm{y}$ 250 empleados) del sector cerámico de la Comunidad Valenciana. La edad media de los encuestados se sitúa en 40.45 años $(\mathrm{DT}=8.23)$ y el $70.8 \%$ son hombres; su antigüedad media en la empresa es de 8.59 años (DT = 6.66) y tienen mayoritariamente estudios superiores (39.9\%) y medios $(41.7 \%)$. El $36.3 \%$ son directivos, el $45.8 \%$ técnicos y el resto operarios $(17.9 \%)$, su contrato es indefinido en un $91.7 \%$ de los casos.

\section{Procedimiento}

Los datos fueron recogidos con la ayuda de una empresa de entrevistadores profesionales, en horario laboral, vía telefónica y web y la participación en el estudio fue totalmente voluntaria. Dado que el principal requisito de la investigación era que los participantes necesitasen compartir su conocimiento para realizar las actividades propias de su trabajo, se entrevistó a cuatro empleados de un mismo equipo, en cada una de las empresas. La muestra se recogió desde octubre de 2010 hasta enero del 2011.

\section{Instrumentos}

El clima se ha medido con dos de las cinco dimensiones de la escala de Anderson y West (1998), orientación a la tarea y apoyo a la innovación, ampliamente utilizadas en la investigación sobre innovación (Hulsheger et al, 2009). La orientación a la tarea también conocida como clima de excelencia describe "una preocupación compartida por lograr la calidad en los resultados del trabajo"(p. 313) y el apoyo a la innovación "la aprobación y la ayuda práctica para introducir nuevas y mejores formas de hacer el trabajo" (West y Farr, 1990, p. 315). La orientación a la tarea se ha evaluado con tres ítems (ejemplo: «En mi equipo/departamento somos capaces de evaluar de forma crítica nuestro potencial para lograr el mejor resultado posible») y el apoyo a la innovación con cuatro (ejemplo: «Mi equipo/departamento siempre está dispuesto a buscar nuevas soluciones»). Los coeficientes de fiabilidad son .74 y .84 , respectivamente.

El compromiso afectivo es una actitud que expresa el vínculo emocional del empleado con su organización. Se evalúa con 4 ítems de la escala de Allen y Meyer (1996) (ejemplo: «Me gustaría continuar el resto de mi carrera profesional en esta organización»). El coeficiente de fiabilidad es .83

El apoyo organizacional se mide con la escala de Eisenberger (Eisenberger et al., 1986) que se centra en cómo valora la organización las contribuciones individuales de los empleados y se preocupa y cuida de su bienestar personal. Se ha evaluado con 4 ítems (ejemplo «Mi organización se preocupa de mi bienestar»). El coeficiente de fiabilidad es .83.

Figura 1. Modelo propuesto

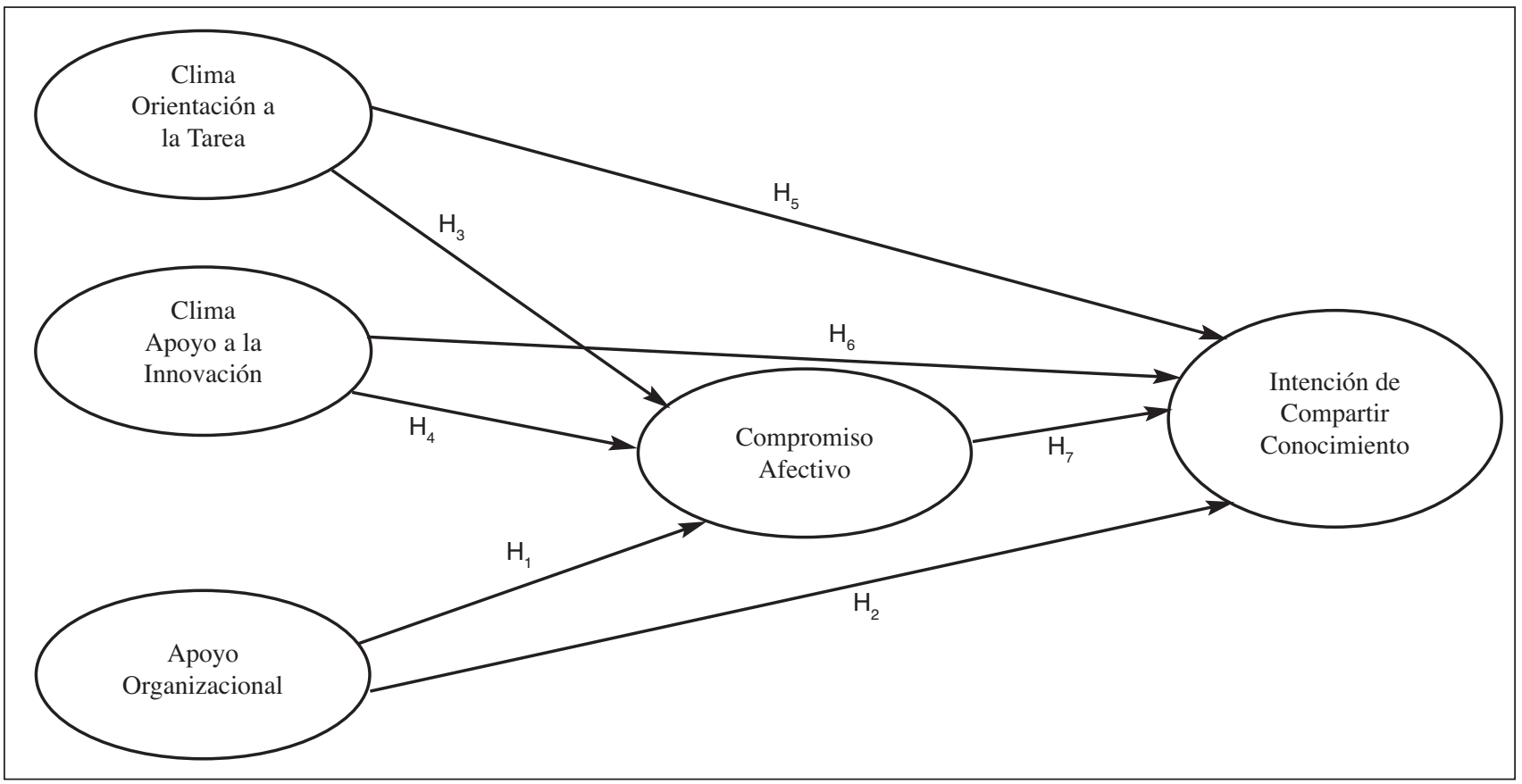


Las respuestas a estos tres instrumentos se recogían en escalas tipo Likert de cinco puntos, donde 1 corresponde a «nada de acuerdo» y 5 a «totalmente de acuerdo».

Por último, para medir la intención de compartir conocimiento se utiliza la escala de Cummings (2004) que se centra en conocer la disposición del empleado a intercambiar información con los compañeros sobre cómo realizar el trabajo, para ayudar y colaborar en la resolución de problemas, desarrollo de nuevas ideas o introducción de procedimientos para mejorar el desempeño. Se han utilizado 5 ítems (ejemplo «Estoy dispuesto a compartir con mis compañeros de equipo/departamento mi conocimiento y experiencias de cómo realizar el trabajo»), con respuestas de escala Likert de cinco puntos (1 «nunca» y 5 «siempre»). El valor de la fiabilidad es igual a .83 .

\section{Análisis de datos}

Como se ha planteado en el objetivo, este artículo analiza las relaciones entre las variables propuestas con un modelo de ecuaciones estructurales. Un modelo de ecuaciones estructurales completo consta de dos partes: el modelo de medida y el modelo de relaciones estructurales (Anderson y Gerbing, 1988; Bollen, 1995; Hair, Anderson, Tatham y Black, 2008; Ruiz, Pardo y San Martín, 2009). El modelo de medida contiene la forma en que cada variable se mide mediante sus indicadores observables, los errores que afectan a las mediciones y las relaciones que se espera encontrar entre los constructos, cuando están relacionados entre sí (Ruiz et al., 2009). Esto aporta información de la fiabilidad y validez de los indicadores como medidas de las variables latentes (Bollen, 1995). El modelo de relaciones estructurales es el modelo a estimar y contiene las relaciones entre las variables y los errores de predicción (Ruiz et al., 2009). Su objetivo es confirmar si las relaciones causales que se especifican en el modelo son consistentes con los datos (Bollen, 1995).

Para evaluar las características de las escalas de medida y la estimación del modelo estructural se ha utilizado el programa estadístico EQS 6.1 (Bentler, 1995) mediante máxima verosimilitud (ML) con estimadores robustos. El ajuste del modelo global se ha evaluado con tres tipos de medidas, de ajuste absoluto, parsimonioso e incremental, porque son complementarias y permiten asegurar el modelo desde distintas perspectivas (Levy y Williams, 2004; Hair et al., 2008). Las medidas de ajuste absoluto analizan el grado de ajuste global del modelo propuesto a los datos. Las medidas de ajuste incremental comparan el ajuste del modelo con el ajuste del modelo nulo (el que asume la ausencia de relaciones entre variables) y finalmente, las medidas de ajuste parsimonioso proporcionan información sobre el número de coeficientes a estimar para lograr un determinado nivel de ajuste y tratando de evitar el sobreajuste con coeficientes innecesarios. Como medidas de ajuste absoluto se ha considerado el valor de la Satorra - Bentler $\chi^{2}$ (chi-cuadrado) y los índices GFI (Goodness of Fit Index), AGFI (Adjusted Goodness of Fit Index), RMSEA (Root Mean Squared Error of Approximation). Para el GFI y el AGFI, se consideran apropiados valores mayores que 0.9. Por su parte, (Browne y Cudeck, 1992) sugieren que un modelo con un índice RMSE igual o menor que .08 indica un ajuste razonable. En cuanto a las medidas incrementales de ajuste, se utilizan los índices BB-NFI (Bentler-Bonett Normed Fit Index) ó BBNNFI (Bentler-Bonett Non-Normed Fit Index). Se consideran apropiados valores mayores que 0.9. Para el análisis de la parsimonia se utiliza el índice NC o chinormada ( $\chi 2$ /g.1.).

En primer lugar, se han verificado las escalas de medida utilizando el Análisis Factorial Confirmatorio y se ha estimado un modelo de medida distinto para cada una de las variables de la investigación, en concreto, el clima compuesto por las dimensiones orientación a la tarea y apoyo a la innovación, el apoyo, el compromiso afectivo y la intención de compartir conocimiento. También se ha comprobado la estructura factorial de segundo orden de la variable clima.

Los resultados del análisis (Tabla 1) indican que los distintos modelos de medida ajustan adecuadamente a

Tabla 1. Índices de bondad de ajuste de los modelos de medida de las variables

\begin{tabular}{|c|c|c|c|c|c|c|c|c|c|c|c|}
\hline & $\chi^{2}$ & $\begin{array}{c}\text { Satorra- } \\
\text { Bentler } \\
\text { Chi-square }\end{array}$ & $\mathrm{gl}^{\mathrm{a}}$ & $\mathrm{p}$ & $\mathrm{NFI}^{\mathrm{b}}$ & $\mathrm{NNFI}^{\mathrm{c}}$ & $\mathrm{RCFI}^{\mathrm{d}}$ & $\mathrm{GFI}^{\mathrm{e}}$ & $\mathrm{AGFI}^{\mathrm{f}}$ & RMSEA $^{\mathrm{g}}$ & $\mathrm{NC}^{\mathrm{h}}$ \\
\hline Clima (Orientación a la tarea) & 20.47 & 20.40 & 18 & .311 & .950 & .990 & .994 & .970 & .940 & .028 & 1.13 \\
\hline Clima (Apoyo a la innovación) & 2.41 & 26.65 & 2 & .264 & .982 & .986 & .995 & .993 & .964 & .045 & 1.33 \\
\hline Apoyo organizacional & 1.80 & 1.58 & 1 & .209 & .988 & .973 & .996 & .995 & .945 & .060 & 1.15 \\
\hline Compromiso afectivo & 1.17 & 1.18 & 1 & .277 & .994 & .994 & .999 & .997 & .965 & .033 & 1.18 \\
\hline \multicolumn{12}{|l|}{ Intención de compartir } \\
\hline conocimiento & 5.34 & 5.15 & 4 & .273 & .979 & .988 & .995 & .988 & .954 & .042 & 1.29 \\
\hline Clima del equipo & 20.95 & 19.71 & 13 & .103 & .933 & .960 & .975 & .965 & .924 & .056 & 1.52 \\
\hline
\end{tabular}

Nota: agl: grados de libertad; ${ }^{\mathrm{b}} \mathrm{NFI} \geq .9 ;{ }^{\mathrm{c}} \mathrm{NNFI} \geq .9 ;{ }^{\mathrm{d}} \mathrm{RCFI} \geq .9 ;{ }^{\mathrm{e}} \mathrm{GFI} \geq .9 ;{ }^{\mathrm{f}} \mathrm{AGFI} \geq .9 ;{ }^{\mathrm{g}} \mathrm{RMSEA} \leq .08 ;{ }^{\mathrm{g}} \mathrm{RMSEA} \leq .05 ;{ }^{\mathrm{h}} \mathrm{NC}$

(valores entre 1 y 2 ) 
los datos para cada una de las variables consideradas. Todos los valores de la Satorra - Bentler $\chi^{2}$ son estadísticamente significativos $(p>.05)$. El comportamiento de todos los índices de ajuste permite afirmar la existencia de una única dimensión para el apoyo organizacional y el compromiso afectivo y de un factor de segundo orden para el clima organizacional.

Además, se ha comprobado la fiabilidad y validez de las escalas (Bagozzi y Yi, 1990; Bagozzi y Phillips, 1982; Fornell y Larcker, 1981). La fiabilidad calculando el coeficiente de fiabilidad compuesto por ser más adecuado que el alpha de Cronbach, ya que no depende del número de atributos asociados a cada concepto (Hair et al., 2008). Como se aprecia en la Tabla 2, el instrumento de medida no muestra problemas de fiabilidad, pues el índice de fiabilidad compuesta es superior para todos los factores al valor recomendado de 0.70 (Fornell y Larcker, 1981) y la varianza extraída es superior o cercana a 0.50 (Tabla 3) (Fornell y Larcker, 1981).

La validez convergente se calculó a través de la significación estadística de las cargas factoriales de todos los indicadores de cada constructo latente. En la Tabla 2 se comprueba que todas las cargas estandarizadas poseen valores superiores a 0.50 y son estadísticamente significativas como se desprende de sus valores $t$ (superiores a 1.96), superando ampliamente los níveles mínimos recomendados (Hair et al., 2008). Además, el coeficiente de Bentler-Bonett, para todos los modelos de medida supera el valor de .90 (ver BB-NFI en Tabla 1) (Bentler y Bollen, 1980). También existe validez discriminante entre las medidas, puesto que como se observa en la Tabla 3, los intervalos de confianza en la estimación de las correlaciones entre cada par de factores no incluyen el 1, lo que indica según Anderson y Gerbing (1988) que existe validez discriminante al

Tabla 2. Fiabilidad y validez convergente del modelo

\begin{tabular}{|c|c|c|c|c|}
\hline Factor & $\begin{array}{l}\text { Indicador } \\
\mathrm{r}\end{array}$ & $\begin{array}{c}\text { Carga } \\
\text { factorial }\end{array}$ & $\begin{array}{l}\text { Valor T } \\
\text { robusto }\end{array}$ & $\begin{array}{l}\text { Fiabilidad } \\
\text { Compuesta }\end{array}$ \\
\hline \multicolumn{5}{|c|}{ Clima - Orientación a la Tarea } \\
\hline & COT1 & $.65^{*}$ & & \multirow{3}{*}{.72} \\
\hline & COT2 & .79 & 4.57 & \\
\hline & COT3 & .59 & 3.76 & \\
\hline \multicolumn{5}{|c|}{ Clima - Apoyo a la innovación } \\
\hline & CAI1 & $.69 *$ & & \multirow{4}{*}{.85} \\
\hline & CAI2 & .62 & 6.94 & \\
\hline & CAI3 & .82 & 9.03 & \\
\hline & CAI4 & .90 & 8.34 & \\
\hline \multirow[t]{4}{*}{ Apoyo organizacional } & AP1 & $.71 *$ & & \multirow{4}{*}{.82} \\
\hline & AP2 & .64 & 8.61 & \\
\hline & AP3 & .85 & 7.45 & \\
\hline & AP4 & .72 & 7.73 & \\
\hline \multicolumn{5}{|l|}{ Compromiso afectivo } \\
\hline & CA1 & $.65^{*}$ & & \multirow{4}{*}{.81} \\
\hline & CA2 & $.61 *$ & 8.64 & \\
\hline & CA3 & .82 & 8.62 & \\
\hline & CA4 & .77 & 7.59 & \\
\hline \multicolumn{5}{|c|}{ Intención de compartir conocimiento } \\
\hline & ICC1 & $.77 *$ & & \multirow{5}{*}{.88} \\
\hline & $\mathrm{ICC} 2$ & .69 & 10.43 & \\
\hline & ICC3 & .75 & 11.62 & \\
\hline & ICC4 & .86 & 8.66 & \\
\hline & ICC5 & .75 & 9.67 & \\
\hline \multicolumn{5}{|l|}{ Clima del equipo } \\
\hline & COT1 & $.72 *$ & & \multirow{7}{*}{$.83 *$} \\
\hline & COT2 & .72 & 6.58 & \\
\hline & COT3 & .58 & 6.02 & \\
\hline & CAI1 & .70 & 10.30 & \\
\hline & CAI2 & .63 & 11.42 & \\
\hline & CAI3 & .82 & 17.95 & \\
\hline & CAI4 & .90 & 19.32 & \\
\hline
\end{tabular}

* parámetro fijado a 1 
Tabla 3. Validez discriminante de las variables

\begin{tabular}{|c|c|c|c|c|c|}
\hline & 1 & 2 & 3 & 4 & 5 \\
\hline Clima-Orientación a la tarea & .46 & .39 & .42 & .15 & .25 \\
\hline Clima-Apoyo a la innovación & $(.31-.41)$ & .59 & .29 & .22 & .17 \\
\hline Apoyo organizacional & $(.59 ;-.72)$ & $(.47-.61)$ & .54 & .32 & .13 \\
\hline Compromiso afectivo & $(.48-.58)$ & $(.53-.63)$ & $(.60-.78)$ & .52 & .19 \\
\hline Intención de compartir conocimiento & $(.46-.55)$ & $(.41-.50)$ & $(.39-.49)$ & $(.46-.55)$ & .59 \\
\hline
\end{tabular}

Nota: En la diagonal aparece el AVE. Sobre la diagonal la varianza compartida. Bajo la diagonal el intervalo de confianza al 95\% para la estimación de la correlación entre factores.

95\% y la varianza promedio extraída para cada factor es superior al cuadrado de la correlación entre cada par de factores (Fornell y Larcker, 1981).

En este punto y con estos resultados se puede concluir que, en conjunto las escalas de medida utilizadas para evaluar el clima (sus dimensiones de orientación a la tarea y de apoyo a la innovación), el apoyo, el compromiso afectivo y las intenciones de compartir conocimiento, presentan validez y consistencia interna, por tanto son idóneas para medir los conceptos teóricos que forman el modelo propuesto.

\section{Resultados}

Una vez confirmadas las medidas, se presentan las medias, desviaciones típicas y los coeficientes de correlación de Pearson entre las variables (Tabla 4). En segundo lugar y para comprobar en qué grado las relaciones entre las variables organizacionales (apoyo y clima) y personales (compromiso afectivo) y las intenciones de compartir conocimiento son consistentes con los datos se plantea el modelo estructural (Figura 1).

En la Tabla 5 se muestran los índices de bondad de ajuste de este modelo y se puede observar que todos los resultados (Satorra - Bentler $\chi^{2}=177.37 ; \mathrm{p}=.165$, $\mathrm{BB}-\mathrm{NFI}=.867, \mathrm{BB}-\mathrm{NNFI}=.982, \mathrm{RCFI}=.985, \mathrm{GFI}=$ $.897, \mathrm{AGFI}=.865$ y RMSEA $=.026)$, indican que el ajuste del modelo es adecuado, pues están dentro de los límites habitualmente aceptados, aunque algunas de las relaciones planteadas no resulten estadísticamente significativas.

A partir de la solución estandarizada recogida en la Figura 2, se pueden extraer los siguientes resultados. En primer lugar, se confirma que el apoyo organizacional se relaciona significativamente con el compromiso afectivo con la organización $(b=.52 ; p<.05)$, coincidiendo con lo planteado en la hipótesis 1 , aunque su relación con las intenciones de compartir conocimiento no alcanza la significación mínima exigida ( $b=-.12 ;$ n.s.), en contra de lo planteado en la hipótesis 2. En segundo lugar, la influencia del clima sobre estos consecuentes depende de la dimensión que se considere. Así, la dimensión apoyo a la innovación muestra relaciones directas y significativas con el compromiso afectivo $(b=.28 ; p<.05)$, en línea con la hipótesis 4 , pero las relaciones con la intención de compartir conocimiento no alcanzan el nivel mínimo de significación $(b=.02 ; \mathrm{n} . \mathrm{s}$.), lo que nos lleva a rechazar la hipótesis 6 . El patrón contrario se observa con la

Tabla 4. Medias, desviaciones típicas, fiabilidades y correlaciones entre las variables

\begin{tabular}{|c|c|c|c|c|c|c|c|c|}
\hline Variables & M & DT & $\alpha$ & 1 & 2 & 3 & 4 & 5 \\
\hline Clima-Orientación a la tarea & 3.99 & .48 & .74 & - & & & & \\
\hline Clima-Apoyo a la innovación & 3.94 & .48 & .84 & $.63 * *$ & - & & & \\
\hline Apoyo organizacional & 3.79 & .66 & .83 & $.65^{* *}$ & $.54 * *$ & - & & \\
\hline Compromiso afectivo & 3.74 & .56 & .83 & $.39 * *$ & $.47 * *$ & $.57 * *$ & - & \\
\hline Intención de compartir conocimiento & 4.06 & .53 & .86 & $.50 * *$ & $.42 * *$ & $.37 * *$ & $.44 * *$ & - \\
\hline
\end{tabular}

Nota: $p \leq .05^{*} ; p \leq .01 * *$

Tabla 5. Índices de bondad de ajuste del modelo estructural propuesto

\begin{tabular}{lccccccccccc}
\hline & $\chi^{2}$ & $\begin{array}{c}\text { Satorra- } \\
\text { Bentler } \\
\text { Chi-square }\end{array}$ & $\mathrm{gl}^{\mathrm{a}}$ & $\mathrm{p}$ & $\mathrm{NFI}^{\mathrm{b}}$ & $\mathrm{NNFI}^{\mathrm{c}}$ & $\mathrm{RCFI}^{\mathrm{d}}$ & $\mathrm{GFI}^{\mathrm{e}}$ & $\mathrm{AGFI}^{\mathrm{f}}$ & $\mathrm{RMSEA}^{\mathrm{g}}$ & $\mathrm{NC}^{\mathrm{h}}$ \\
\hline Modelo Propuesto & 185.43 & 177.37 & 160 & .165 & .867 & .982 & .985 & .897 & .865 & .026 & 1.11 \\
\hline
\end{tabular}

Nota: ${ }^{\mathrm{a} g l}=$ grados de libertad; ${ }^{\mathrm{b}} \mathrm{NFI} \geq .9 ; \mathrm{cNFI} \geq .9 ;{ }^{\mathrm{d}} \mathrm{RCFI} \geq .9 ; \mathrm{eFI} \geq .9 ; \mathrm{f} \mathrm{AFI} \geq .9 ;{ }^{\mathrm{g}} \mathrm{RMSEA} \leq .08 ;{ }^{\mathrm{g}} \mathrm{RMSEA}$

$\leq .05 ;{ }^{\mathrm{h}} \mathrm{NC}$ (valores entre 1 y 2 ) 
Figura 2. Relación del clima y apoyo organizacional con la intención de compartir conocimiento a través del compromiso afectivo

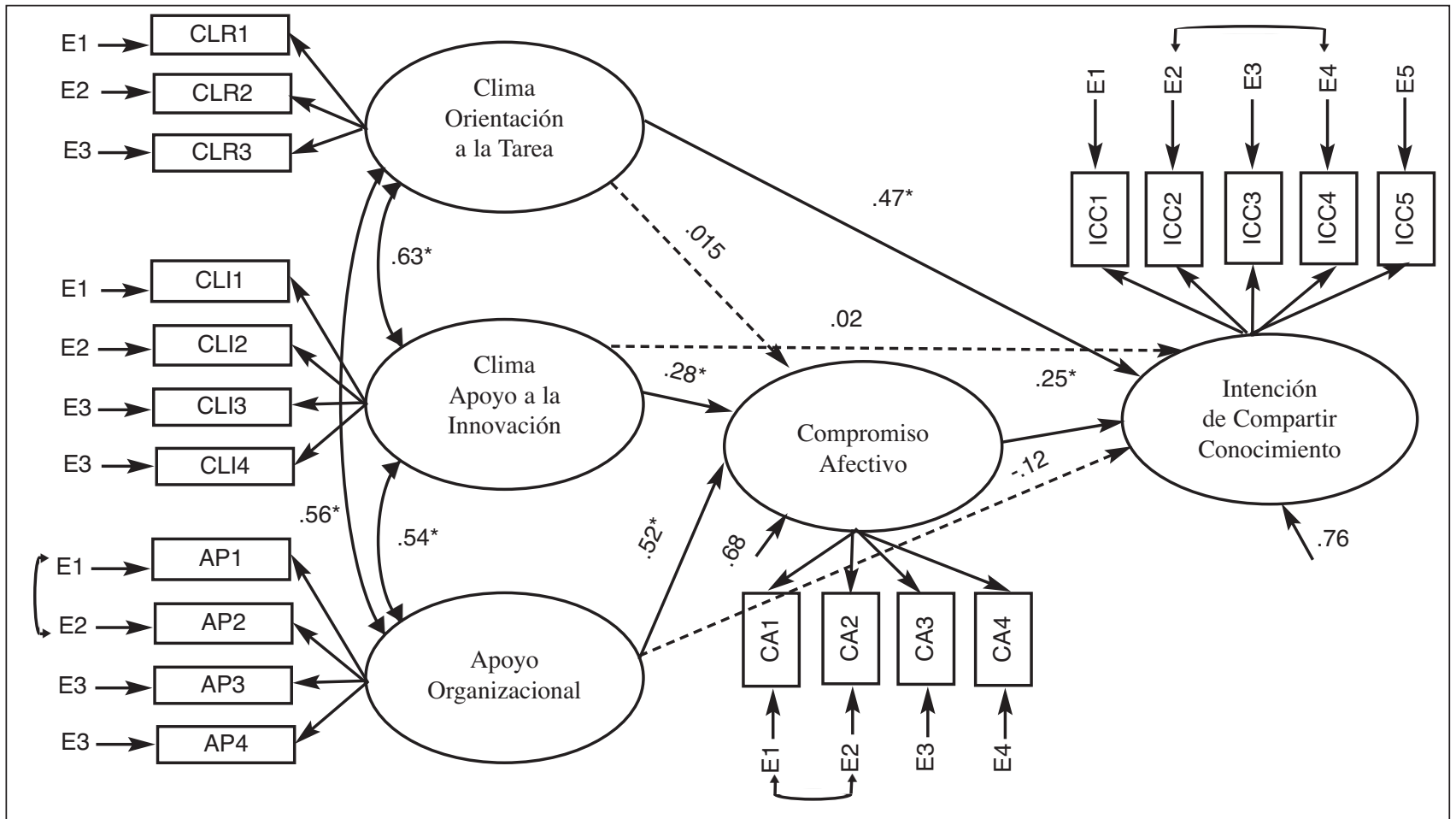

*Coeficiente significativo al $95 \%$

dimensión orientación a la tarea, ya que no presenta relaciones significativas con el compromiso afectivo $(b=.01 ; \mathrm{n} . \mathrm{s}$.), en contra de la hipótesis 3 , pero sí con las intenciones de compartir conocimiento $(b=.47 ; p<$ $.05)$, lo que apoya la hipótesis 5. Por último, tal y como planteaba la hipótesis 7 , el compromiso afectivo se relaciona positivamente con las intenciones que tienen los empleados de compartir su conocimiento con sus colegas $(b=.25 ; p<.05)$. El modelo propuesto explica el $42.4 \%$ de la varianza de la intención de compartir conocimiento.

\section{Discusión}

La forma de gestionar el conocimiento de los empleados se revela como un aspecto clave de la innovación y el éxito de las empresas de ahí que los estudios se hayan multiplicado en los últimos años (Wang y Noe, 2010; Foss et al., 2010). No obstante, la investigación todavía plantea dudas acerca de los antecedentes que impulsan a un empleado a compartir lo que sabe con sus compañeros. Para avanzar en esta cuestión, este artículo ha analizado a través de ecuaciones estructurales el peso de tres variables, organizacionales y personales, en las intenciones de compartir conocimiento. Dado que no se ha encontrado bibliografía que avale el modelo planteado, se ha partido de la investigación que analiza los antecedentes organizacionales de las conductas de desempeño extra-rol, ya que así consideran algunos autores las conductas de compartir conocimiento (Yu y Chu, 2007).

Los resultados de los análisis efectuados han permitido corroborar buena parte de las hipótesis planteadas. Concretamente y como esperaba, el clima organizacional y específicamente la dimensión orientación a la tarea se relaciona directamente con la intención de los empleados de compartir lo que saben con sus compañeros. Este resultado coincide, por ejemplo, con los de Zarraga y Bonache (2003) quienes observan que un clima orientado y abierto a nuevas ideas y mejoras influye positivamente en las conductas de compartir su conocimiento y también con otros de fuera de nuestro contexto (Lee y Choi, 2003; Lin y Lee, 2006).

Las otras dos dimensiones organizacionales consideradas, el apoyo y el clima de apoyo a la innovación, aunque se relacionan una a una con las intenciones de compartir conocimiento, al poner a prueba del modelo conjunto se observa que esta relación actúa de forma indirecta, a través del papel del compromiso afectivo. Según esto, aunque los empleados que se sienten más apoyados muestran más intenciones de compartir lo que saben con sus compañeros, en línea con otros trabajos (p. ej. Bartol et al., 2009; Cabrera et al., 2006; Kulkarni et al., 2006), esta relación directa desaparece al considerar su grado de compromiso afectivo con la organización, de forma que el apoyo aumenta el compromiso y es esto lo que a su vez aumenta las intenciones de compartir conocimiento. Estos datos están en la línea con los trabajos que destacan el importante papel 
del apoyo organizacional en el compromiso afectivo (p. ej. Rhoades, Eisenberger y Armeli, 2001; Riggle, Edmondson y Hansen, 2009) y también con los que consideran el compromiso como variable intermedia entre determinadas prácticas de recursos humanos de alta implicación y los resultados organizacionales (p. ej. Cabrera y Cabrera, 2005).

Los resultados parecen claros, un clima organizacional orientado a la tarea (que busca la excelencia, la mejor forma de hacer las cosas, etc.) influye directamente en las intenciones de compartir el conocimiento con los compañeros, probablemente porque la existencia de normas y procedimientos claros al respecto induce a los empleados seguirlos y a actuar consecuentemente. A la vez, sentirse apoyado por la organización, tanto en la realización de tareas innovadoras como en el trabajo en general, aumenta el compromiso afectivo con la organización y es esto lo que potencia a su vez que los empleados tengan más intenciones de compartir.

Antes de finalizar, señalar algunas limitaciones del estudio. Aunque se han realizado análisis estructurales que permiten hablar de relaciones causales (Ruiz et al., 2009), se trata de un estudio de carácter transversal, cuyos resultados convendría corroborar con datos longitudinales. Además, se ha llevado a cabo en un sector, el cerámico y una comunidad, la valenciana, con unas características muy específicas, lo que puede reducir las posibilidades de generalizar sus resultados. Además, el $57.1 \%$ de las empresas analizadas ha experimentado una reducción de su facturación en los tres últimos años, aspecto que también habría que considerar en futuros estudios por si estuviera afectando a los datos. No obstante y a pesar de lo anterior, creemos que el trabajo hace una contribución significativa porque, como se ha visto a lo largo artículo, buena parte de los estudios sobre gestión del conocimiento se han realizado en empresas vinculadas a las nuevas tecnologías y en países orientales. En este sentido, este trabajo permite afirmar que se confirman los patrones observados con otras muestras y en contextos diferentes.

En posteriores estudios además de superar estas limitaciones sería interesante evaluar no sólo las intenciones y ampliarlo a las conductas de compartir conocimiento, como hacen por ejemplo Kim y Lee (2006). Como se sabe, actitudes y conductas no siempre están tan relacionadas como se espera (Fife-Schaw, Sheeran y Norman, 2007) y pueden tener otros antecedentes, diferentes de los considerados aquí. Por último, habría que analizar también qué dimensiones del apoyo y del clima resultan más eficaces para que los empleados compartan lo que saben con sus compañeros. Por ejemplo, qué es más importante, el apoyo de los compañeros o el de los superiores (p. ej. Ng y Sorensen, 2008); que se dé sólo apoyo emocional o es también necesario un determinado nivel de apoyo instrumental (Semmer et al., 2008).

Dada la complejidad que entrañan los conceptos de apoyo y clima organizacional, en futuros estudios convendría incorporar nuevas dimensiones e ir matizando su contribución. Lo mismo sucede con el compromiso con la organización. En este trabajo sólo se ha considerado su dimensión afectiva, sin embargo, diferentes trabajos muestran que las dimensiones normativa y de continuidad con la organización, también se relacionan con diferentes actitudes organizacionales (Han et al., 2010) de ahí su interés.

A pesar de todo lo anterior y dada la escasez de estudios empíricos en el ámbito de la gestión del conocimiento similares, creemos que este trabajo permite clarificar dos aspectos. En primer lugar, contribuye a aclarar de forma empírica las razones que impulsan a los empleados a compartir lo que saben con sus compañeros. En segundo, analiza de forma conjunta variables organizacionales y personales, al proponer un modelo que incluye relaciones directas e indirectas, como se ha sugerido (Cabrera et al., 2006). Destacar además que, a pesar del reducido número de variables antecedentes consideradas, se consigue explicar un porcentaje de varianza de la variable consecuente importante.

Por último y desde un punto de vista aplicado, podemos afirmar que si las organizaciones quieren que sus empleados compartan con sus compañeros sus conocimientos, habilidades, experiencia, etc., es fundamental que se sientan apoyados y que reciban instrucciones claras sobre el trabajo que deben realizar y los objetivos últimos que se quiere alcanzar. Según los resultados de este estudio si se hace así, se aumentarán las intenciones de compartir lo que se sabe, lo que redundará en una mejor gestión del conocimiento organizacional y, en definitiva, en mayores éxitos. 


\section{Extended Summary}

Knowledge is a strategic organizational resource in a competitive and dynamic economy. Organizations' competitive advantage increasingly depends on effective knowledge management (e.g. Gagné, 2009). Research has shown that knowledge sharing among employees is a core process related to in-firm innovation (Camelo, Garcia, \& Sousa, 2010) and organizational learning (Foss, Husted, \& Michailova, 2010). However, employees do not share their knowledge spontaneously and the reason why individual employees decide to share their knowledge with others is currently not understood. More research is needed to examine how individual and organizational factors enhance knowledge sharing (Wang \& Noe, 2010). Therefore, the goal of the present study is to contribute to the understanding of several individual and organizational factors determining employees' knowledgesharing intentions; organizational support, psychological climate and organizational commitment.

Only a few empirical studies have investigated the effect of perceived organizational support on knowledge-sharing intentions. It appears, however, that organizational support is likely to influence knowledge-sharing (Bartol, Liu, Zeng, \& Wu, 2009; Cabrera, Collins, and Salgado, 2006; Kulkarni, Ravindran, \& Freeze, 2006). Similarly, a positive climate is related to innovation (Hulsheger, Anderson, \& Salgado, 2009) and more specifically, to knowledge-sharing intentions and behaviors (Kim \& Lee, 2006; Lee \& Choi, 2003, Lin \& Lee, 2006; Zarraga \& Bonache, 2003). Finally, employees who are committed to their organization are willing to perform extrarole behaviors and to engage in knowledge-sharing behaviors (Ardichvili, 2008; Cabrera et al., 2006; Han, Chiang, \& Chang; 2010; Lin, 2007).

Furthermore, recent research has found a positive relationship between organizational support and climate and attitudes toward organization and sharing knowledge intentions through individual factors (e. g. Bock, Zmud, Kim \& Lee, 2005; Tohidinia \& Mosakhani, 2010). Based on this idea, this exploratory study proposes an integrated model that investigates the influence of perceived organizational support and psychological climate on the individuals' knowledgesharing intentions, directly and indirectly through employees' affective commitment toward the organization (Figure 1).

\section{Method}

The present data was collected from 168 employees from 42 medium-sized firms in the tile industry ( 42 work groups). Roughly $70.8 \%$ were male, the average age was 40.45 years $(\mathrm{SD}=8.23)$ and members had an average of 8.59 years $(\mathrm{SD}=6.66)$ of company experi- ence. In the sample, $39.9 \%$ had university degrees and $41.7 \%$ had completed secondary education. Top and middle managers accounted for $36.3 \%$, while $45.8 \%$ were expert technicians and $17.9 \%$ were workers.

Psychological climate was measured using two dimensions from the Team Inventory Questionnaire by Anderson and West (1998). Three and four items were used to measure each of the two dimensions respectively: task orientation and support for innovation ('This team is always moving toward the development of new answers' and 'Does the team continually monitor its own performance in order to achieve the highest standards?'. The alphas were .74 for task orientation and .84 for support for innovation. Affective commitment was assessed using four items from Allen and Meyer scale (1996). One sample item was 'I would be very happy to spend the rest of my career with this organization'. Reliability of the scale was .83. To measure perceived organizational support, we employed four items from Eisenberger, Huntington, Hutchison, and Sowa (1986) scale. One sample item was 'my organization really cares about my well-being'. The scale yielded a reliability of .83 . Finally, knowledge-sharing intention was assessed with a five items scale adapted from Cummnings (2004). One sample item was 'I am willing to share my knowledge and experience, on how to perform my job, with my group of work'. The reliability of the scale was .83. The answers were on a 5-point Likert type scale, from 1 ('totally disagree', or 'never') to 5 ('totally agree', or 'always').

\section{Results}

The analysis of the structural equation model provides some interesting results (see Figure 2); first, organizational support is a critical antecedent of affective commitment (H1) but it does not have a direct effect on employees' knowledge-sharing intentions (H2); second, the influence of psychological climate on this consequent depends on the dimension considered: on the one hand, the dimension of support for innovation shows a direct and significant relationship to affective commitment (H4), but it is not significant enough regarding knowledge-sharing intention (H6). On the other hand, task orientation dimension does not show any significant relationship to affective commitment (H3), but it is significant to knowledge-sharing intentions (H5). Finally, and as expected, affective commitment is positive related to employees' knowledge-sharing intentions. Specifically, $53.1 \%$ of the affective commitment variance is explained by organizational support and the support for innovation of the climate dimension. Moreover, the proposed model explains $42.4 \%$ of the knowledge-sharing intention variance. 


\section{Discussion}

This exploratory research analyzes the influence of perceived organizational support and psychological climate on knowledge-sharing intentions and examines the role of affective commitment among antecedent and consequent variables. As expected, task orientation directly influences the intention of the employees to share their knowledge with their work group. These results fit with Zarraga and Bonache (2003), who found that an open climate oriented to new ideas and continuous improvement influences positively on knowledge-sharing intention. However, although the other two organizational dimensions considered, organizational support and support for innovation, do not influence directly on knowledge-sharing intention, they do indirectly through the employees' commitment to their organization. It appears that employees who perceived support from their organization tend to engage more in knowledge-sharing behaviors, but the effect is washed out once affective commitment enters the model. This fact matches up with other research that highlights the important role of organizational support on affective commitment (e.g., Rhoades, Eisenberger, \& Armeli, 2001; Riggle, Edmondson, \& Hansen, 2009), and those which show the mediation role of affective commitment among some human resources practices and organizational outcomes (e.g. Cabrera \& Cabrera, 2005).

The results of the study clearly report that a task-oriented climate (looking for excellence) influences directly on employees' knowledge-sharing intentions, while feeling supported not only in innovative tasks but also at work in general, increases affective commitment towards the organization and enhances employees' knowledge intention.

Before ending, some limitations should be considered in drawing conclusions from our results; a crosssectional study was conducted and our sample was small and restricted to one sector, and consequently does not provide a basis for testing the generalization of the findings. However, we believe that the present findings contribute to the knowledge-sharing literature because the majority of previous research has been conducted in technological firms and Chinese cultures.

Future research needs to assess both knowledgesharing intentions and behaviors, as investigated by Kim and Lee (2006), because other antecedents may be at work, in addition to those already considered. Finally, more research is needed to clarify which support and climate dimensions are more effective for encouraging knowledge-sharing among employees. For example, what is more important, organizational or co-worker support (e.g. Ng and Sorensen, 2008)? Or emotional or instrumental support (Semmer et al., 2008)? Due to the complexity of both concepts, in future research, it would be interesting to continue to study their contribution and to analyze the influence of other dimensions of organizational commitment (Han et al., 2010).

Our findings contribute in knowledge-sharing literature in two respects: First, they clarify empirically the antecedent factors that explain why individuals decide to share their knowledge with others. Second, the proposed model considers both individual and organizational factors and its relationship in an integrated model. Despite the reduced number of factors considered, only four, an important amount of knowledgesharing intentions variance has been explained.

Several implications for organizations can be drawn from the finding in our results. First, organizational support is critical for the success of knowledge-sharing initiatives, so organizations should encourage managers to provide the necessary support and orientation for encouraging knowledge-sharing among employees and increasing individuals' commitment towards the organization.

\section{Referencias}

Allen, N. J. y Meyer, J. P. (1996). Affective, continuance, and normative commitment to the organization: An examination of construct validity. Journal of Vocational Behavior, 49, 252-276.

Alpkan, L. (2010). Organizational support for intrapreneurship and its interaction with human capital to enhance innovative performance. Management Decision, 48, 732.

Anderson, J. C. y Gerbing, D. W. (1988). Structural equation modeling in practice: A review and recommended twostep approach. Psychological Bulletin, 103, 411-423.

Anderson, N. R. y West, M. A. (1998). Measuring climate for work group innovation: Development and validation of the team climate inventory. Journal of Organizational Behavior, 19, 235-258.

Ardichvili, A. (2008). Learning and knowledge sharing in virtual communities of practice: Motivators, barriers, and enablers. Advances in Developing Human Resources, 10, 541-554. doi:10.1177/1523422308319536

Bagozzi, R. P. y Yi, Y. J. (1989). On the use of structural equation models in experimental-designs. Journal of Marketing Research, 26, 271-284.

Bagozzi, R. P. y Yi, Y. J. (1990). New perspectives on the application of structural equation models. 1990 Ama Educators Proceedings: Enhancing Knowledge Development in Marketing, 1, 282-282.

Bagozzi, R. P. y Phillips, L. W. (1982). Representing and testing organizational theories: A holistic construal. Administrative Science Quarterly, 27(3), 459-489. Retrieved from http://search.ebscohost.com/login.aspx?direct= trueyd $b=$ buhyAN $=4014456$ yite $=$ ehost-liveyscope $=$ site

Bartol, K. M., Liu, W., Zeng, X. y Wu, K. (2009). Social exchange and knowledge sharing among knowledge workers: The moderating role of perceived job security. Management and Organization Review, 5, 223-240. doi:10.1111/j.1740-8784.2009.00146.x 
Bentler, P. M. (1995). EQS structural equations program manual Encino, CA: Multivariate Software, Inc.

Bentler, P. M. y Bonett, D. G. (1980). Significance test and goodness-of-fit in the analysis of covariance structures. Psychological Bulletin, 88, 588-606.

Bock, G. W., Zmud, R. W., Kim, Y. G. y Lee, J. N. (2005). Behavioral intention formation in knowledge sharing: Examining the roles of extrinsic motivators, social-psychological forces, and organizational climate. Mis Quarterly, 29, 87-111.

Bollen, K. A. (1995). Structural equation models that are nonlinear in latent variables: A least-squares estimator. Sociological Methodology, 25, 223-251.

Browne, M. W. y Cudeck, R. (1992). Alternative ways of assessing model fit. Sociological Methods y Research, 21, 230-258.

Cabrera, A., Collins, W. C. y Salgado, J. F. (2006). Determinants of individual engagement in knowledge sharing. International Journal of Human Resource Management, 17, 245-264. doi:10.1080/09585190500404614

Cabrera, E. F. y Cabrera, A. (2005). Fostering knowledge sharing through people management practices. International Journal of Human Resource Management, 16, 720735. doi:10.1080/09585190500083020

Camelo, C., Garcia, J. y Sousa, E. (2010). Knowledge sharing: Enablers and its influence on innovation. Cuadernos de Economia y Direccion de la Empresa, 42, 113-150.

Carr, J. Z., Schmidt, A. M., Ford, J. K. y DeShon, R. P. (2003). Climate perceptions matter: A meta-analytic path analysis relating molar climate, cognitive and affective states, and individual level work outcomes. Journal of Applied Psychology, 88, 605-619. doi:10.1037/00219010.88.4.605

Chen, Z., Eisenberger, R., Johnson, K. M., Sucharski, I. L. y Aselage, J. (2009). Perceived organizational support and extra-role performance: Which leads to which? Journal of Social Psychology, 149, 119-124.

Choi, J. N. (2007). Change-oriented organizational citizenship behavior: Effects of work environment characteristics and intervening psychological processes. Journal of Organizational Behavior, 28, 467-484. doi:10.1002/job. 433 ER

Cropanzano, R. y Mitchell, M. S. (2005). Social exchange theory: An interdisciplinary review. Journal of Management, 31, 874-900. doi:10.1177/0149206305279602

Cummings, J. N. (2004). Work groups, structural diversity, and knowledge sharing in a global organization. Management Science, 50, 352-364. doi:10.1287/mnsc.1030 .0134

D'Amato, A. y Zijlstra, F. R. H. (2008). Psychological climate and individual factors as antecedents of work outcomes. European Journal of Work and Organizational Psychology, 17, 33-54. doi:10.1080/13594320701307420 ER

Darolia, C. R., Kumari, P. y Darolia, S. (January 2010). Perceived organizational support, work motivation, and organizational commitment as determinants of job performance. Journal of the Indian Academy of Applied Psychology, 36, 69-78.

Dawley, D. (2010). Perceived organizational support and turnover intention: The mediating effects of personal sacrifice and job fit. Journal of Social Psychology, 150, 238.

Edwards, M. R. y Peccei, R. (2010). Perceived organizational support, organizational identification, and employee outcomes testing a simultaneous multifoci model. Journal of Personnel Psychology, 9, 17-26. doi:10.1027/1866-5888/a000007

Eisenberger, R., Armeli, S., Rexwinkel, B., Lynch, P. D. y Rhoades, L. (2001). Reciprocation of perceived organizational support. Journal of Applied Psychology, 86, 42-51.

Eisenberger, R., Huntington, R., Hutchison, S. y Sowa, D. (1986). Perceived organizational support. Journal of Applied Psychology, 71, 500-507.

Fife-Schaw, C., Sheeran, P. y Norman, P. (2007). Simulating behaviour change interventions based on the theory of planned behaviour: Impacts on intention and action. British Journal of Social Psychology, 46, 43-68. Doi: 10.1348/14466605X85906

Filipova, A. A. (2011). Relationships among ethical climates, perceived organizational support, and intent-toleave for licensed nurses in skilled nursing facilities. Journal of Applied Gerontology, 30, 44-66.

Fornell, C. y Larcker, D. F. (1981). Evaluating structural equation models with unobservable variables and measurement error. Journal of Marketing Research (JMR), 18, 39-50.

Foss, N. J., Husted, K. y Michailova, S. (2010). Governing knowledge sharing in organizations: Levels of analysis, governance mechanisms, and research directions. Journal of Management Studies, 47, 455-482 . doi:10.1111/j.14676486.2009.00870.x

Golden, T. D. y Raghuram, S. (2010). Teleworker knowledge sharing and the role of altered relational and technological interactions. Journal of Organizational Behavior, 31, 1061-1085. doi:10.1002/job.652

Gouldner,A.W. (1960). The norm of reciprocity - a preliminary statement. American Sociological Review, 25, 161-178.

Guerrero, S. y Herrbach, O. (2009). Manager organizational commitment: A question of support or image? International Journal of Human Resource Management, 20(7), 1536-1553. doi:10.1080/09585190902983496

Hair, J. F., Anderson, R. E., Tatham, R. L. y Black, W. C. (2008). Análisis multivariante. Madrid: Prentice Hall.

Han, T., Chiang, H. y Chang, A. (2010). Employee participation in decision making, psychological ownership and knowledge sharing: Mediating role of organizational commitment in taiwanese high-tech organizations. International Journal of Human Resource Management, 21(12), 2218-2233. doi:10.1080/09585192.2010.509625

Hulsheger, U. R., Anderson, N. y Salgado, J. F. (2009). Team-level predictors of innovation at work: A comprehensive meta-analysis spanning three decades of research. Journal of Applied Psychology, 94(5), 11281145. doi:10.1037/a0015978 ER 
Kalman, M. E. (1999) 'The Effects of Organizational Commitment and Expected Outcomes on the Motivation to Share Discretionary Information in a Collaborative Database: Communication Dilemmas and Other Serious Games'. Unpublished dissertation. University of Southern California.

Kim, S. y Lee, H. (2006). The impact of organizational context and information technology on employee knowledge-sharing capabilities. Public Administration Review, 66(3), 370-385. doi:10.1111/j.1540-6210.2006.00595.x

Kulkarni, U. R., Ravindran, S. y Freeze, R. (2006). A knowledge management success model: Theoretical development and empirical validation. Journal of Management Information Systems, 23(3), 309-347. doi:10.2753/MIS 0742-1222230311

Lee, H. y Choi, B. (2003). Knowledge management enablers, processes, and organizational performance: An integrative view and empirical examination. Journal of Management Information Systems, 20(1), 179-228.

Lester, S. W., Meglino, B. M. y Korsgaard, M. A. (2008). The role of other orientation in organizational citizenship behavior. Journal of Organizational Behavior, 29(6), 829-841. doi:10.1002/job.504

Levy, P. E. y Williams, J. R. (2004). The social context of performance appraisal: A review and framework for the future. Journal of Management, 30(6), 881-905. doi: 10.1016/j.jm.2004.06.005

Lin y Lee. (2006). Effects of socio-technical factors on organizational intention to encourage knowledge sharing. Management Decision, 44(1), 74.

Lin, C. (2007). To share or not to share: Modeling knowledge sharing using exchange ideology as a moderator. Personnel Review, 36(3), 457-475. doi:10.1108/00483480710731374

Liu, Y. (2009). Perceived organizational support and expatriate organizational citizenship behavior: The mediating role of affective commitment towards the parent company. Personnel Review, 38(3), 307.

MacKain, S. J., Myers, B., Ostapiej, L. y Newman, R. A. (2010). Job satisfaction among psychologists working in state prisons. Criminal Justice and Behavior, 37(3), 306318. doi:10.1177/0093854809357420

Meyer, J. P., Stanley, D. J., Herscovitch, L. y Topolnytsky, L. (2002). Affective, continuance, and normative commitment to the organization: A meta-analysis of antecedents, correlates, and consequences. Journal of Vocational Behavior, 61(1), 20-52. doi:10.1006/jvbe.2001.1842

$\mathrm{Ng}$, T. W. H. y Sorensen, K. L. (2008). Toward a further understanding of the relationships between perceptions of support and work attitudes - A meta-analysis. Group y Organization Management, 33(3), 243-268. doi:10.1177/1059601107313307

Ostroff, C. y Schmitt, N. (1993). Configurations of organizational-effectiveness and efficiency. Academy of Management Journal, 36(6), 1345-1361.

Parker, C. P., Baltes, B. B., Young, S. A., Huff, J. W., Altmann, R. A., Lacost, H. A., et al. (2003). Relationships between psychological climate perceptions and work out- comes: A meta-analytic review. Journal of Organizational Behavior, 24(4), 389-416. doi:10.1002/job.198

Panaccio, A. y Vandenberghe, C. (2009). Perceived organizational support, organizational commitment and psychological well-being: A longitudinal study. Journal of Vocational Behavior, 75(2), 224-236. doi:DOI: 10.1016/ j.jvb.2009.06.002

Pazy, A. y Ganzach, Y. (2009). Pay contingency and the effects of perceived organizational and supervisor support on performance and commitment. Journal of Management, doi:10.1177/0149206307312505

Rhoades, L. y Eisenberger, R. (2002). Perceived organizational support: A review of the literature. Journal of Applied Psychology, 87(4), 698-714. doi:10.1037//00219010.87.4.698

Rhoades, L., Eisenberger, R. y Armeli, S. (2001). Affective commitment to the organization: The contribution of perceived organizational support. Journal of Applied Psychology, 86(5), 825-836. doi:10.1037//0021-9010.86.5. 825

Riggle, R. J., Edmondson, D. R. y Hansen, J. D. (2009). A meta-analysis of the relationship between perceived organizational support and job outcomes: 20 years of research. Journal of Business Research, 62(10), 10271030. doi:10.1016/j.jbusres.2008.05.003

Riketta, M. (2002). Attitudinal organizational commitment and job performance: A meta-analysis. Journal of Organizational Behavior, 23(3), 257-266. doi:10.1002/job.141

Rousseau, V. y Aube, C. (2010). Team self-managing behaviors and team effectiveness: The moderating effect of task routineness. Group \& Organization Management, 35(6), 751-781.

Ruiz, M. A., Pardo, A. y San Martín, R. (2009). Modelos de ecuaciones estructurales. Papeles Del Psicólogo: Revista Del Colegio Oficial De Psicólogos, 31(1), 34-45.

Semmer, N. K., Elfereing, A., Jacobshagen, N. y Perrot, T. (2008). The emocional meanig of instrumental social support. International Journal of Stress Management, 15(3), 235-51. doi: 10.1037-5245/15.3.235.

Shelton, P. M., Waite, A. M. y Makela, C. J. (2010). Highly effective teams: A relational analysis of group potency and perceived organizational support. Advances in Developing Human Resources, 12(1), 93-114. doi:10. $1177 / 1523422310365665$

Simonin, B. L. (2004). An empirical investigation of the process of knowledge transfer in international strategic alliances. Journal of International Business Studies, 35 (5), 407.

Sluss, D. M., Klimchak, M. y Holmes, J. J. (2008). Perceived organizational support as a mediator between relational exchange and organizational identification. Journal of Vocational Behavior, 73(3), 457-464. doi: 10.1016/j.jvb.2008.09.001

Tohidinia, Z. y Mosakhani, M. (2010). Knowledge sharing behaviour and its predictors. Industrial Management y Data Systems, 110(3-4), 611-631. doi:10.1108/0263-5571 011039052 
Tremblay, M., Cloutier, J., Simard, G., Chenevert, D. y Vandenberghe, C. (2010). The role of HRM practices, procedural justice, organizational support and trust in organizational commitment and in-role and extra-role performance. International Journal of Human Resource Management, 21(3), 405-433. doi:10.1080/09585190903549056

Tsai, M. y Cheng, N. (2010). Programmer perceptions of knowledge-sharing behavior under social cognitive theory. Expert Systems with Applications, 37(12), 8479-8485. doi:10.1016/j.eswa.2010.05.029

Webster, J. R. y Adams, G. A. (2010). Organizational sup- port and contract fulfillment as moderators of the relationship between preferred work status and performance. Journal of Business and Psychology, 25(1), 131-138. doi:10.1007/s10869-009-9135-z

Yu, C. y Chu, T. (2007). Exploring knowledge contribution from an OCB perspective. Information \& Management, 44(3), 321-331. doi:10.1016/j.im.2007.03.002

Zarraga, C. y Bonache, J. (2003). Assessing the team environment for knowledge sharing: An empirical analysis. International Journal of Human Resource Management, 14(7), 1227-1245. doi:10.1080/0958519032 000114282

Manuscrito recibido: 22/10/2011

Revisión recibida: 06/12/2011

Aceptado: 09/12/2011 